\title{
The Use of Predictive Analytics to Shift the Culture of Academic Advising Toward a Focus on Student Success
}

\author{
Paul R. Bowdre, Ed.D. \\ Office of Assessment and Institutional Effectiveness \\ Oakland City University \\ 138 N. Lucretia Street \\ Oakland City, IN 47660 \\ USA
}

\begin{abstract}
This paper explores the use of data through a predictive analytical approach to shift the current culture of academic advising from one of compliance to one that focuses on student learning and success in all areas of an educational experience.As educational institutions demonstrate an ever-increasing reliance on the use of data to make evidencebased decisions, the use of much of the same data can be used to develop an evidence-based profile of student performance. Advisors using analytics can predict future outcomes and proactively guide students to make betterdecisions regarding their selection of an academic major or coursework pathways, improving a student's likelihood of attaining a degree and consequent success in a given field. Due to the technological advances in the areas of educational data mining and the use of analytics, the capacity to guide students using rational, evidence-based information is now a possibility. This paper sets the stage for the use of predictive analytics that can influence a cultural shift in academic advising while offering caution that educational institutions must assess the quality of the data decisions that are being made.
\end{abstract}

Keywords: Predictive analytics, learning analytics, big data, evidence-based decisions, academic advising, targeted advising, student success

\section{Introduction}

Data has been identified as critical to the success of any modern organization," and it has been linked to a growing number of changes in the education industry. According to Banihashem, Aliabadi, Ardakani, Delaver,and Ahmadabadi (2018), data plays a phenomenal role in the education sector by providing reliable and real-time insights on areas to focus on and allocate resources. The researchers go on to explain that data has been depicted as one of the most critical elements in the education industry that cannot be touched or seen, and today we often hear such elements referred to as big data and analytics (Banihashem et al., 2018). Chen and Upah (2018) also explore the significance of big data in the education industry. According to the authors, data is pivotal in the provision of better outcomes for students and educators. Using data, administrators, and other stakeholders can determine why students succeed or fail particular subjects. Moreover, it is the best way of illustrating why some concepts are not easily learned in schools, and yet there are those that are easily understood by the learners (Chen \&Upah, 2018).

The emergence of the big data concept has revolutionized the modern business environment. Today, businesses rely on big data to make evidence-based decisions. Thus, Taylor-Sakyi (2016) explains that big data is a combination of both structured and unstructured sets of data. Moreover, the aim of using the data is to provide insights into some of the areas not traditionally explored by statistics (Taylor-Sakyi, 2016). There are three key parameters that drive the use of big data: technology, analysis, and mythology. Taylor-Sakyi (216) argues that the tremendous change in the adoption of technology explains why big data has become common and sought-after concept. The need to effectively utilize computing power and data algorithms has made it necessary to embrace big data among most businesses (Zeide, 2016). At the same time, there is a general belief that big data is the source of business intelligence, and its multi-dimensional approach makes it even appealing to the organizations in the market (Taylor-Sakyi, 2016). In this regard, the evolution of big data has somewhat replaced the model-driven science as organizations embrace the data-driven science model.

The emergence of big data can be traced back to early 2011 (Alsghaier, Akour, Shehabat \& Aldiabat, 2017). The continued development and implementation of the concept in the business environment have not only given some entities a competitive advantage but also improved the general position of the economy as a whole. Furthermore, the beneficiaries of big data are not only individual businesses but also governments and other supporting stakeholders like the community. Using a case study methodology, Alsghaier (2017) explores the evolution and significance of big data. According to the researcher, big data has the ability to spur revolutionary transformations in every sector. A vital role that has been played by big data in the education industry is the evaluation of student and institutional performance. 
Banihashem et al. (2108) explain that the emergence of big data in the learning environment has led to the development of learning analytics, which is a combination of pedagogy, artificial intelligence, machine learning, and learning technologies. In addition, learning analytics is defined as a collection and use of data within the context of educationthe data involves the behavior, capacity, and skills of the learners (Lester, Klein, Rangwala \& Johri, 2017; Banihashem et al., 2018). Therefore, learning analytics is correlated to data-driven techniques aimed at offering optimized solutions in the educational environment (Nunn, Avella, Kanai \& Kebritchi, 2016). Generally, the rise and implementation of big data concept have significantly improved the decision making the ability of educational stakeholders in the society by providing data-driven insights which are hailed as reliable, less costly, and optimized.

\section{Predictive Analytics}

Predictive analytics relies on data, statistics, and machine learning to identify the likelihood of future occurrences. The primary objective is to go beyond the current into the future - provide the best assessment of what will happen in the future (Ballinger, 2018; Brecht \& Burnett, 2019). Although the concept of predictive analytics has been around, its time has come as more organizations turn to predictive analytics to increase their competitive advantage in the market. The key reasons why the technology has gained momentum include the increase in the volume of data, presence of cheaper and reliable computers, availability of easy to use software components, and increased pressure in the market with the entry of more businesses (Ekowo \& Palmer, 2016; Gardner \& Brooks, 2018). In their study, Chen and Upa $\mathrm{h}(2018)$ argue that predictive analytics is critical in the education industry as it provides a superior way of analyzing relationships and trends of such variables as behavior, performance, and use of resources. Using statistical tools, schools can adequately predict future outcomes if certain variables are taken into consideration (Chen \&Upah, 2018). For example, if additional resources are invested in games, schools can predict if games will improve the socialization of the students or their performance in various games championship. Therefore, the use of predictive analytics enhances the planning process of education organizations.

Predictive analytics builds upon the proactive key concepts of learning analytics that include access to the data and the application of properly implemented processes. According to Gardner and Brooks (2018), predictive modeling has become a central framework in learning analytics research. The researchers argue that the increased usage of learning technologies such as open online courses have made it possible for institutions to use predictive learning (Gardner \& Brooks, 2018). However, the researchers point out that although predictive analytics is portrayed as a vital concept, it is not fully understood by the industry players - hence, the data mining and model selection gap. Today, organizations are turning to the use of predictive analytics to solve many emerging challenges (Klempin, Grant \& Ramos, 2018). For example, businesses are finding analytical tools vital in dealing with fraud, optimization of marketing campaigns, improvement of internal operations, and reduction of risks. A combination of various analytic methods can aid organizations in improving pattern detection and avoid criminals. Similarly, marketing agencies are benefitting from the use of predictive analytics (Ekowo \& Palmer, 2016). Using available data and predictive analytic models, organizations are able to determine the behavior of their clients and promote cross-selling. Moreover, the predictive analytic framework enhances the ability of companies to forecast inventory and manage resources (Baer \& Carmean, 2019).

The foundation of predictive analytics is data, predictive models, and executive contributions (Ekowo \& Palmer, 2016). At the start, a problem is identified within an entity, and data related to the problem is collected. Using the preferred model, the future consequence of a specific variable is assessed. Based on the findings, the executive is expected to react to solve the problem or improve on a positive outcome. According to Brecht and Burnett (2019), predictive analytics can involve multiple outcomes. In their study, the researchers used nine predictive variables to forecast the performance of students. Although not all of these variables directly impacted the predictive model, they provided a clue on the significance of a multi-dimensional approach in the assessment of the behavior of the people. The significance of multi-dimensional models is also supported by Banumathi and Aloysius (2017), who explain the importance of combining predictive analytics and statistical modeling. Three data models can be used in big data: descriptive, prescriptive, and predictive. In an education setting, descriptive analytics is aligned towards the use of raw data, predictive tries to decipher the future probabilities of the data, and prescriptive information is given to the students (Banumathi \& Aloysius, 2017). Basically, predictive analytics follows different models in cross-examining current data to future events.

\section{Predictive Analytics in Education}

The education sector is one of the main beneficiaries of the rapid changes in technology in society. In his article, Michael Horn evaluates the use of technology in education. According to his analysis, technology is viewed as a key learning and teaching enabler and has given numerous students the opportunity to enhance their grades. Moreover, the 
significance attached to technology has intensified the efforts to equip learning institutions with better and recent technological tools(Horn, 2017). Therefore, the use of technology increases in the education sector so does the demand to leverage predictive analytics. The significance of data analytics in the education industry is depicted by Chen and Upah (Chen \& Upah, 2018). According to the researchers, the applications and functionalities of big data provide a wide range of opportunities to the stakeholders. For this reason, governments across the world have launched plans and roadmaps to support the development of big data in public and private entities aligned with the delivery of educational practices. According to Phillips (2013), the improvement of the technological infrastructures in education provides a framework for institutions in the sector to leverage on the concept of big data. Phillips goes on and argues that the primary determinant of the success of big data is the infrastructure laid out by learning and teaching institutions. In this regard, if the technology is not properly integrated its benefits will not be attained in the long run (Phillips, 2013). The same argument is supported by Parnell, Jones, Wesaw, and Brooks (n.d.) who review the role of data analytics in student success. According to Parnell et al. (n.d.), leveraging on big data to enhance success among students requires meticulous planning and investments to have in place the required tools to collect and analyze data. Taking into consideration this argument, it can be concluded that continued investment in technology in the education industry has immensely facilitated the development and implementation of data analytics and predictive analytics in the education sector.

A publication by the American Council on Education and Center for Education Attainment and Innovation (2015) shows that the higher education is at a transition stage, whereby modern systems are beginning to take the center stage. The publication goes on to offer some of the emerging solutions to problems affecting the quality and reliability of the education industry. These solutions include predictive analytics and collaborative learning - all leading to personalized learning and development. Essentially, the focus is placed on the use of data to make critical decisions and boost the performance of learners. Moreover, the transition from the traditional system of decision making to the modern intelligent structures is bound to offer a future positive outlook as it spearheads the implementation of personalized systems of teaching and learning. A systematic literature review conducted by Bagriyanik (2017) finds that a personalized learning environment plays an integral role in allowing students to acquire knowledge and skills at their own pace. As opposed to the overall classroom setting where students follow the same model of learning, a personalized setting is uniquely designed, taking into consideration the strengths and weaknesses of an individual (Bagriyanik, 2017). Therefore, the development of predictive analytics is pivotal in the establishment of tailored learning models in higher institutions of learning. The significance of personalized learning is also exhibited by Ballinger (2018). According to the author, the society has a responsibility of fostering success for everyone - no child should be left behind. In this regard, every dream matters, and institutions should use the available tools to attain every child's dream [6]. The solutions to enhancing learning and, more particularly, personalized learning are shared by both Ballinger (2018) and Banihashem et al.(2018). The authors depict that adopting a culture of learning and harnessing the power of predictive analytics is vital for success in institutions of higher learning. Ballinger (2018) argues the power of data cannot be underestimated as it provides deeper integral insights into the performance of students. Moreover, the use of big data allows educators to develop models measuring the growth of student performance and predicting their future outcomes if certain metrics such as access to resources are twerked.

In their systematic literature review, Viberg, Hatakka, Bälter,and Mavroudi (2018) argue that the role of learning analytics in higher education is yet to fully be exploited. Although many institutions have implemented technologies in their teaching and learning models and improved asynchronous data sharing, they are yet to fully utilize learning analytics to improve the quality of their education (Viberg et al., 2018). Therefore, there is a huge gap that remains unaddressed in as far as data analytics is concerned and it is time to leverage on the existing capacities to improve educational programs to suit the needs of the learners. The same argument is shared by Mangaroska and Giannakos(2018) who also use a systematic literature review to assess the role of learning analytics. According to these researchers, there is a synergy between learning analytics and learning design. The modern system of learning is highly dependent on prior and projected future outcomes. Therefore, policymakers, educators, and other-stakeholders widely implement data-driven decision making (Mangaroska \& Giannakos, 2018). The aim is to ensure that models, processes, and systems put in place are at tandem with the current and future quality standards.

Hence, data analytics will remain to be a pivotal area of concern for institutions of higher learning. For this Lester, Klein, Rangwala, and Johri (2018) assert that the incorporation of predictive analytics in student support and learning designs as a growing area that should be quickly implemented in higher education. However, there is a need to appropriately plan for the implementation of predictive analytics. This is because it requires significant changes, process redesign, and re-imagination of key roles of institutions of learning (Lester et al., 2018). 
Typically, while the researchers acknowledge the significance of predictive learning, they are quick to point out that its implementation should be meticulous to attain the expected results.

\section{Predictive Analytics Advising Role}

The advising role of predictive analytics is captured by a study done by Klempin, Grant, and Ramos(2018). In this study, the researchers investigate the perspective of college personnel involved in the consideration, launch, and use of predictive analytics for targeted advising. The authors found that predictive analytics is phenomenal in re-shaping the direction of education in society. Through predictive analytics, students and advisors are able to interact more closely. Moreover, educational institutions are able to provide crucial advice to the advisors and students on areas to put more focus to succeed. However, the researchers note that despite the substantial promise of predictive analytics in learning institutions, there needs to be a greater examination to address such concerns as validity, interpretation, and ethics (Klempin, Grant \& Ramos, 2018). The issue of predictive analytics and personalization is also discussed by Liu, Bartimote-Aufflick, Pardo, and Bridgeman (2017) whereby they argue that although predictive analytics has been acknowledged as a significant way of dealing with educational issues, it remains minimally used in the higher education. According to the researchers, before the use of big data, institutions relied on technology mediation to increase the support student attain while participating in learning experiences. However, the emergence of big data has led to the development of predictive algorithms to achieve the same improvements. Thus, predictive analytics offers a comprehensive understanding of how students work and what factors to consider in improving their performance (Liu, Bartimote-Aufflick, Pardo \& Bridgeman, 2017). Student success is largely dependent on tailoring their learning experiences and ensuring that minimal costs are incurred in the educational programs.

The increasing cost of higher education has necessitated the implementation of predictive analytics in higher learning. According to Liu, Bartimote-Aufflick, Pardo, and Bridgeman (2017), there is a need for students and parents to reduce the length of stay in institutions of higher learning by finding learning strategies that will harness success within the shortest time. Similarly, higher learning institutions do not want to incur high costs offering programs that may not be successful in the long run. In this regard, institutions are having a keen interest in predictive analytics to assess the level of dropouts, course uptakes, and graduation rates to make vital decisions on whether to continue offering certain courses or not. Further, on their end, students have to evaluate the success rate of their programs and make decisions on whether their options are viable or not (Liu, Bartimote-Aufflick, Pardo \& Bridgeman, 2017). The significance of predictive analytics is closely associated with that of learning analytics, according to Patwa, Seetharaman, Sreekumar,and Srinivas (2018). According to these authors, using predictive analytics, higher education organizations are able to use data to provide insights on market trends. Therefore, management can be able to target students who are more likely to enroll in their programs.

Higher education institutions are able to immensely reduce their cost of operations owing to the avoidance of wastages and targeting the right students (Patwa, Seetharaman, Sreekumar \& Srinivas, 2018). This is a capacity brought about by the use of predictive analytics in an education setting. Patwa et al. (2018) go on to depict that the significance of predictive analytics can also be felt by the students. Higher education organizations are able to take advantage of the information collected to forecast the performance of students. These institutions can better analyze whether students are at risk and help them perform by improving their weak areas. In lieu of the same argument, Finnie, Fricker, Bozkurt, Poirier,and Pavlic (2017)depict that predictive analytics offers a mechanism to develop early alerts and intrusive advising techniques in higher education. In their study, Finnie et al. (2017) used two phases of investigation to determine the role of predictive analytics (Phase 1: the development of a predictive model and determination of its role in education; Phase 2: analysis of the specific advising interventions offered to students). The researchers found that there is a strong correlation between student performance and predictive analytics (Finnie et al., 2017). Predictive analytic models offer a baseline for decision making. Hence, educators have a data-driven model to justify their pieces of advice in institutions of learning.

The Educause Center for Analysis and Research (ECAR) (2017) segments predictive analytics in education into learning analytics and academic analytics. The focus of learning analytics is to aid the learners and the learning process. On the other hand, the aim of academic analytics is to use data to improve business operations (ECAR, 2017).

The combination of the two sub-categories of analytics is vital in higher learning as they focus on both the students and the institutions to attain long term success. Predictive analytics proves fundamental in promoting completion efficiency, personalizing advice, and integrating career advice in higher education. Over the last decade, governments across the world have continually re-evaluated their curriculum models. The improvement of curricular and co-curricular support systems is geared towards supporting student success(Roberts, Howell, Seaman \& Gibson, 2016). The decisions to improve or change a curriculum are based on existing data and projections into the future (ECAR, 2017). Hence, 
predictive analytic models have played an integral role in innovative and cost-effective advising strategies employed by institutions of learning to improve student degree completion and career readiness. The improvement of degree completion rates is a top priority in any institution of higher learning.

According to Daniel (2015), the cost of higher learning is largely influenced by the completion rates. Hence, organizations in the industry have resort to the use of predictive analytics to improve their program completion rates as cost mitigation and quality improvement measure (Daniel, 2015). Kharade and Wagh (2016) also explain that the emergence of big data analytics and learning analytics have offered institutions of learning a lifeline to compete in the market. Using these concepts, educational entities are able to deliver uniquely tailored services to their customers and, in the end, increase their profit margins (Kharade \& Wagh, 2016). In this regard, the focus of higher institutions of learning is to pinpoint any factors that may hinder their operations and work towards avoidance or mitigation of the risks using data-driven insights. Furthermore, the increased government focus on degree productivity and increased pressure on higher education leaders to improve degree completion rates have led to the implementation of predictive analytics as an important approach of aiding institutions to attain their goals (Upah, 2016). At the same time, students and parents are beginning to consider such elements as the time to degree and average debt load. These factors are pushing parents to ask more questions on completion rates and student success. Therefore, the focus is no longer placed on enrolling in institutions of learning, rather on the time of completion and the success of the students in these institutions(Steele, 2018). There are a host of methods higher institutions can implement to influence student success, but one that stands out is the use of predictive analytical tools (Liu, Bartimote-Aufflick, Pardo \& Bridgeman, 2017). According to Liu et al. (2018), having more direct control over the performance of students is integral in advising them on which areas to improve. Moreover, it is important to individually evaluate students and identify their weaknesses and advise them on what courses to pursue to attain their dreams. Hence, the role of predictive analytics in advising cannot be underrated in higher learning institutions.

The advising challenges vary in various institutions as each of these entities has a different environment. Thus, there is no single-fit-all solution to their problems. It is at this point that each institution needs to develop its unique model of operation and leverage on predictive analytics to find out what factors affect learners. There are different advising models that can be relied upon by learning institutions (Viberg et al., 2018). For example, institutions can rely on personalized models or general models to manage their operations. The choice of a model is dependent on the challenges faced by the learners. In a situation where learners exhibit similar challenges, a general model can be deployed. However, if learners exhibit unique challenges, a personalized model is advisable, and this is where the role of predictive analytics manifests. According to Steele (2018), the two main issues affecting the education industry are technology and data analytics. According to the researcher, the combination of technology and data analytics has transformed the education sector. As compared to the past century, the modern education system immensely relies on data-driven decisions (Steele, 2018). For example, institutions have to enroll students based on their capabilities, interests, and overall performance and not only on their ability to pay for education programs. In fact, this is the model used by some of the largest institutions of learning like Harvard University, which has recognized the significance of data analytics in predicting the performance of students. Moreover, institutions have transformed their teaching models from the traditional general teaching approaches to new personalized techniques (Upah, 2016). The implementation of data analytics in the education system is supported by the widespread acceptance of learning analytics among students. However, according to Roberts, Howell, Seaman, and Gibson (2016), there is a need to guide students in decision making and consideration of ethical issues associated with learning analytics in the education industry.

\section{Predictive Analytics and Culture Shift}

Higher institutions of learning are widely using predictive analytics for culture shifts. By analyzing past and present experiences, these institutions are able to make decisions to shift or not shift to embrace a new model(Tierney \& Lanford, 2017). For centuries, most institutions of higher learning embraced the centralized form of leadership that gave senior management the autonomy of making all decisions. However, decades of data analysis have shown that this method of leadership is no longer viable. Therefore, today higher education organizations have decentralized their decision-making processes to allow junior managers who interact with students on a daily basis to make decisions. Hence, the shift from the traditional leadership model is largely informed by the use of analytics in decision making. Institutions of higher learning are adjusting their teaching models to implement a collaborative and adaptive learning model (Zeide, 2016). This is a transition from the traditional classroom setting. The need to reduce inefficiencies and improve student performance has facilitated a radical change in the industry. Generally, the use of analytics has led to numerous changes in higher education. Organizations in the industry no longer dictate the market conditions; rather, take the direction that analytics dictate. Apart from the institutions of learning, parents and students have moved away from the traditional concept of gauging performance to the modern system characterized by analytics (Baer \& 
Carmean, 2019). Parents have to consider the strengths and weaknesses of their children and aid them in making decisions on the choice of careers or university programs.

\section{Conclusion}

Higher learning institutions are turning to predictive analytics to increase their performance and competitive advantage in the industry. In order for these institutions to differentiate themselves and remain competitive at the same time, they need to pinpoint and understand the needs of their students. However, reliance on traditional methods of survey only limits their decisions as these methods only offer insights after an occurrence. Therefore, they cannot be used adequately when dealing with real-time data like grades that provide future insights. The solution to this problem lies in the use of predictive analytics to aid in the identification of completion rates, student success, and personalized learning models in higher learning. There are numerous applications of predictive analytics in institutions of higher learning, including efficient student advising, adaptive learning, and management of enrollment. Similarly, predictive analytics has facilitated a culture shift towards efficiency and reliability.

\section{References}

Alsghaier, H. (2017). The importance of big data analytics in business: A case study. American Journal of Software Engineering and Applications, 6(4), 111-115. https://doi:10.11648/j.ajsea.20170604.12

Alsghaier, H., Akour, M., Shehabat, I., \& Aldiabat, S. (2017). The impact of big data analytics on business competitiveness. Proceedings of the New Trends in Information Technology (NTIT-2017).

American Council on Education \& Center for Education Attainment and Innovation. (2015). Moving the needle on predictive analytics.https://www.acenet.edu/Documents/Quick-Hits-Predictive-Analytics.pdf

Baer, L., \&Carmean, C. (2019). An Analytics Handbook: Moving From Evidence to Impact. Ann Arbor, MI: Society for College and University Planning.

Bagriyanik, S. (2017). Personal learning environments: A systematic literature review based on study keywords using thematic analysis. New Trends and Issues Proceedings on Humanities and Social Sciences, 4(4), 122-130. https://doi:10.18844/prosoc.v4i4.2603

Ballinger, M. (2018). Advising and predictive analytics: The HT2 of student success (High-Tech; High-Touch). Community College Leadership for the 21St Century, 2-4.

Banihashem, S., Aliabadi, K., Ardakani, S., Delaver, A., \& Ahmadabadi, M. (2018). Learning analytics: A Systematic literature review. Interdiscip Journal of Virtual Learning in Medical Sciences, 9(2), e63024. https://doi:10.5812/ijvlms.6302410.5812/ijvlms.63024

Banumathi, S., \& Aloysius, A. (2017). Predictive analytics concepts in big data - A survey. International Journal of Advanced Research in Computer Science, 8(8), 27-30. https://doi:10.26483/ijarcs.v8i8.4628

Brecht, A., \& Burnett, D. (2019). Advising student-athletes for success: Predicting the academic success and persistence of collegiate student-athletes. NACADA Journal, 39(1), 49-59. https://doi:10.12930/nacada-17-044

Chen, Y., \& Upah, S. (2018). Data analytics and STEM student success: The impact of predictive analytics-informed academic advising among undeclared first-year engineering students. Journal of College Student Retention: Research, Theory \& Practice, https://doi:10.1177/1521025118772307

Daniel, B. (2015). Big Data and analytics in higher education: Opportunities and challenges. British Journal of Educational Technology, 46(5), 904-920. https://doi:10.1111/bjet.12230

Educause Center for Analysis and Research (ECAR). (2017). The predictive learning analytics revolution: Leveraging learning data for student success. ECAR Working Group Paper. https://library.educause.edu/ /media/files/library/2015/10/ewg1510-pdf

Ekowo, M., \& Palmer, I. (2016). The promise and peril of predictive analytics in higher education. A Landscape Analysis.http://www.lonestar.edu/multimedia/The\%20Promise\%20and\%20Peril\%20of\%20Predictive\%20Anal ytics\%20in\%20Higher\%20Education.pdf

Finnie, R., Fricker, T., Bozkurt, E., Poirier, W., \& Pavlic, D. (2017). Using predictive modelling to inform early alert and intrusive advising interventions and improve retention. Toronto: Higher Education Quality Council of Ontario.http://www.heqco.ca/SiteCollectionDocuments/Final\%20Mohawk\%20Predictive\%20Modelling.pdf

Gardner, J., \& Brooks, C. (2018). Evaluating predictive models of student success: Closing the methodological gap. Journal of Learning Analytics, 5(2), 105-125. https://doi:10.18608/jla.2018.52.7

Horn, M. (2017). New research answers whether technology is good or bad for learning. https://www.forbes.com/sites/michaelhorn/2017/11/14/new-research-answers-whether-technology-is-good-orbad-for-learning/\#4589ad1d19d7

Kharade, B., \& Wagh, K. (2016). Educational data mining and learning analytics in higher education. International Journal of Computer Applications (0975 - 8887). 
https://www.researchgate.net/publication/303765189_Data_Analytics_in_Educational_Management_System

Klempin, S., Grant, M., \& Ramos, M. (2018). Practitioner perspectives on the use of predictive analytics in targeted advising for college students. CCRC Working Paper No. 103.

https://ccrc.tc.columbia.edu/media/k2/attachments/practitioner-perspectives-predictive-analytics-targetedadvising.pdf

Lester, J., Klein, C., Rangwala, H., \& Johri, A. (2017). Learning analytics in higher education. ASHE Higher Education Report, 43(5), 9-135. https://doi:10.1002/aehe.20121

Liu, D., Bartimote-Aufflick, K., Pardo, A., \& Bridgeman, A. (2017). Data-driven personalization of student learning support in higher education. Cham, Switzerland: Springer International Publishing.

Mangaroska, K., \& Giannakos, M. (2018). Learning analytics for learning design: A systematic literature review of analytics-driven design to enhance learning. IEEE Transactions on Learning Technologies, 1-1. https://doi:10.1109/ttt.2018.2868673

Nunn, S., Avella, J., Kanai, T., \& Kebritchi, M. (2016). Learning analytics methods, benefits, and challenges in higher education: A systematic literature review. Online Learning, 20(2). https://doi:10.24059/olj.v20i2.790

Parnell, A., Jones, D., Wesaw, A., \& Brooks, C. (n.d.). Institutions' use of data and analytics for student success: Results from a national landscape analysis. https://www.naspa.org/images/uploads/main/DATA2018_DOWNLOAD.pdf

Patwa, N., Seetharaman, A., Sreekumar, K., \& Srinivas, P. (2018). Learning analytics: enhancing the quality of higher education. Research Journal of Economics, 2(2), 1-7.

Phillips, E. (2013). Improving advising using technology and data analytics. Change: The Magazine of Higher Learning, 45(1), 48-55. https://doi:10.1080/00091383.2013.749151

Roberts, L., Howell, J., Seaman, K., \& Gibson, D. (2016). Student attitudes toward learning analytics in higher education: "The Fitbit Version of the Learning World."Frontiers in Psychology, 7. https://doi:10.3389/fpsyg.2016.01959

Steele, G. (2018). Student success: Academic advising, student learning data, and technology. New Directions for Higher Education, 2018(184), 59-68. https://doi:10.1002/he.20303

Taylor-Sakyi, K. (2016). Big data: Understanding big data. https://www.researchgate.net/publication/291229189_Big_Data_Understanding_Big_Data

Tierney, W., \& Lanford, M. (2017). Institutional culture in higher education. Encyclopedia of International Higher Education Systems and Institutions, 1-9. https://doi:10.1007/978-94-017-9553-1_544-1

Upah, S. (2016). The impact of predictive analytics based advising on the selection and change of major among first year, first-term students in engineering. Graduate Theses and Dissertations, 1-163.

Viberg, O., Hatakka, M., Bälter, O., \& Mavroudi, A. (2018). The current landscape of learning analytics in higher education. Computers in Human Behavior, 89, 98-110. https://doi:10.1016/j.chb.2018.07.027

Zeide, E. (2016). 19 Times Data analysis empowered students and schools: Which Students Succeed and why? Future of Privacy Forum.https://fpf.org/wp-content/uploads/2016/03/Final_19Times-Data_Mar2016-1.pdf 